This postprint is published in:

Nonlinear analysis: real world applications, Volume 58, April 2021, art. 103217 [20 pp.]

DOI: 10.1016/j.nonrwa.2020.103217

\title{
ROBIN DOUBLE-PHASE PROBLEMS WITH SINGULAR AND SUPERLINEAR TERMS
}

\author{
N.S. PAPAGEORGIOU, V.D. RĂDULESCU, AND D.D. REPOVŠ
}

\begin{abstract}
We consider a nonlinear Robin problem driven by the sum of $p$-Laplacian and $q$ Laplacian (i.e. the $(p, q)$-equation). In the reaction there are competing effects of a singular term and a parametric perturbation $\lambda f(z, x)$, which is Carathéodory and $(p-1)$-superlinear at $x \in \mathbb{R}$, without satisfying the Ambrosetti-Rabinowitz condition. Using variational tools, together with truncation and comparison techniques, we prove a bifurcation-type result describing the changes in the set of positive solutions as the parameter $\lambda>0$ varies.
\end{abstract}

\section{INTRODUCTION}

Let $\Omega \subseteq \mathbb{R}^{N}$ be a bounded domain with a $C^{2}$-boundary $\partial \Omega$. In this paper, we study the following nonlinear Robin problem

$$
\left\{\begin{array}{c}
-\Delta_{p} u(z)-\Delta_{q} u(z)+\xi(z) u(z)^{p-1}=u(z)^{-\gamma}+\lambda f(z, u(z)) \text { in } \Omega, \\
\frac{\partial u}{\partial n_{p q}}+\beta(z) u^{p-1}=0 \text { on } \partial \Omega, u>0, \lambda>0,0<\gamma<1,1<q<p .
\end{array}\right\}
$$

For every $r \in(1, \infty)$, we denote by $\Delta_{r}$ the $r$-Laplace differential operator defined by

$$
\Delta_{r} u=\operatorname{div}\left(|D u|^{r-2} D u\right) \text { for all } u \in W^{1, r}(\Omega) .
$$

The differential operator of $\left(P_{\lambda}\right)$ is the sum of $p$-Laplacian and $q$-Laplacian. Such an operator is not homogeneous and it appears in the mathematical models of various physical processes. We mention the works of Cherfils \& Ilyasov [2] (reaction-diffusion systems) and Zhikov [22] (elasticity theory). The potential function $\xi \in L^{\infty}(\Omega)$ satisfies $\xi(z) \geqslant 0$ for almost all $z \in \Omega$. In the reaction (the right-hand side of $\left.\left(P_{\lambda}\right)\right)$, we have the combined effects of two nonlinearities of different nature. One nonlinearity is the singular term $u^{-\gamma}$ and the other nonlinearity is the parametric term $\lambda f(z, x)$, where $f(z, x)$ is a Carathéodory function (that is, for all $x \in \mathbb{R}$, the mapping $z \mapsto f(z, x)$ is measurable and for almost all $z \in \Omega$, the mapping $x \mapsto f(z, x)$ is continuous), which exhibits $(p-1)$ superlinear growth near $+\infty$ but without satisfying the usual in such cases Ambrosetti-Rabinowitz condition (the AR-condition for short). In the boundary condition, $\frac{\partial u}{\partial n_{p q}}$ denotes the conormal derivative corresponding to the $(p, q)$-Laplace differential operator. Then according to the nonlinear Green's identity (see Gasinski \& Papageorgiou [3, p. 210]), we have

$$
\frac{\partial u}{\partial n_{p q}}=\left(|D u|^{p-2} D u+|D u|^{q-2} D u, n\right) \text { for all } u \in C^{1}(\bar{\Omega}),
$$

with $n(\cdot)$ being the outward unit normal on $\partial \Omega$. The boundary coefficient $\beta \in C^{0, \alpha}(\partial \Omega)$ (with $0<\alpha<1)$ satisfies $\beta(z) \geqslant 0$ for all $z \in \partial \Omega$.

In the past, nonlinear singular problems were studied only in the context of Dirichlet equations driven by the $p$-Laplacian (a homogeneous differential operator). We mention the works of Giacomoni, Schindler \& Takač [6], Papageorgiou, Rădulescu \& Repovš [11, 12], Papageorgiou \& Smyrlis [17], Papageorgiou \& Winkert [18], and Perera \& Zhang [20]. Nonlinear elliptic problems with unbalanced growth have been studied recently by Papageorgiou, Rădulescu and Repovš $[13,14,16]$. Double-phase transonic flow problems with variable growth have been considered by Bahrouni,

Key words and phrases. Nonhomogeneous differential operator, nonlinear regularity theory, truncation, strong comparison principle, positive solutions

2010 Mathematics Subject Classification: 35J75, 35J92, 35P30. 
Rădulescu and Repovš [1]. A comprehensive study of semilinear singular problems can be found in the book of Ghergu \& Rădulescu [5].

Using variational methods based on the critical point theory together with suitable truncation and comparison techniques, we prove a bifurcation type result, describing in a precise way the dependence of the set of positive solutions of $\left(P_{\lambda}\right)$ on the parameter. So, we produce a critical parameter value $\lambda^{*}>0$ such that for all $\lambda \in\left(0, \lambda^{*}\right)$, problem $\left(P_{\lambda}\right)$ has at least two positive solutions, for $\lambda=\lambda^{*}$ problem $\left(P_{\lambda}\right)$ has at least one positive solution and for $\lambda>\lambda^{*}$ there are no positive solutions for problem $\left(P_{\lambda}\right)$.

\section{Mathematical BaCKGRound AND hypotheses}

Let $X$ be a Banach space. By $X^{*}$ we denote the topological dual of $X$. Given $\varphi \in C^{1}(X, \mathbb{R})$, we say that $\varphi(\cdot)$ satisfies the "C-condition", if the following property holds

"Every sequence $\left\{u_{n}\right\}_{n \geqslant 1} \subseteq X$ such that $\left\{\varphi\left(u_{n}\right)\right\}_{n \geqslant 1} \subseteq \mathbb{R}$ is bounded and $\left(1+\left\|u_{n}\right\|\right) \varphi^{\prime}\left(u_{n}\right) \rightarrow 0$ in $X^{*}$ as $n \rightarrow \infty$, admits a strongly convergent subsequence."

This is a compactness type condition on the functional $\varphi$, which leads to the minimax theory of the critical values of $\varphi(\cdot)$.

The two main spaces in the analysis of problem $\left(P_{\lambda}\right)$ are the Sobolev space $W^{1, p}(\Omega)$ and the Banach space $C^{1}(\bar{\Omega})$. By $\|\cdot\|$ we denote the norm on the Sobolev space $W^{1, p}(\Omega)$. We have

$$
\|u\|=\left[\|u\|_{p}^{p}+\|D u\|_{p}^{p}\right]^{\frac{1}{p}} \text { for all } u \in W^{1, p}(\Omega)
$$

The Banach space $C^{1}(\bar{\Omega})$ is ordered with positive (order) cone given by

$$
C_{+}=\left\{u \in C^{1}(\bar{\Omega}): u(z) \geqslant 0 \text { for all } z \in \bar{\Omega}\right\} .
$$

This cone has a nonempty interior

$$
D_{+}=\left\{u \in C_{+}: u(z)>0 \text { for all } z \in \bar{\Omega}\right\} .
$$

We will also consider another order cone (closed convex cone) in $C^{1}(\bar{\Omega})$, namely the cone

$$
\hat{C}_{+}=\left\{u \in C^{1}(\bar{\Omega}): u(z) \geqslant 0 \text { for all } z \in \bar{\Omega},\left.\frac{\partial u}{\partial n}\right|_{\partial \Omega \cap u^{-1}(0)} \leqslant 0\right\} .
$$

This cone has a nonempty interior

$$
\operatorname{int} \hat{C}_{+}=\left\{u \in C^{1}(\bar{\Omega}): u(z)>0 \text { for all } z \in \Omega,\left.\frac{\partial u}{\partial n}\right|_{\partial \Omega \cap u^{-1}(0)}<0\right\} .
$$

To take care of the Robin boundary condition, we will also use the "boundary" Lebesgue spaces $L^{q}(\partial \Omega)(1 \leqslant q \leqslant \infty)$. More precisely, on $\partial \Omega$ we consider the $(N-1)$-dimensional Hausdorff (surface) measure $\sigma(\cdot)$. Using this measure on $\partial \Omega$ we can define in the usual way the Lebesgue spaces $L^{q}(\partial \Omega)$ $(1 \leqslant q \leqslant \infty)$. We know that there exists a continuous, linear map $\gamma_{0}: W^{1, p}(\Omega) \rightarrow L^{p}(\partial \Omega)$, known as the "trace map" such that

$$
\gamma_{0}(u)=\left.u\right|_{\partial \Omega} \text { for all } u \in W^{1, p}(\Omega) \cap C(\bar{\Omega}) .
$$

So, the trace map extends the notion of boundary values to all Sobolev functions. We have

$$
\operatorname{im} \gamma_{0}=W^{\frac{1}{p^{\prime}}, p}(\partial \Omega)\left(\frac{1}{p}+\frac{1}{p^{\prime}}=1\right) \text { and } \operatorname{ker} \gamma_{0}=W_{0}^{1, p}(\Omega) .
$$

The trace map $\gamma_{0}$ is compact into $L^{q}(\partial \Omega)$ for all $q \in\left[1, \frac{(N-1) p}{N-p}\right)$ if $N>p$ and into $L^{q}(\partial \Omega)$ for all $q \geqslant 1$ if $p \geqslant N$. In the sequel, for the sake of notational simplicity, we drop the use of the trace map $\gamma_{0}(\cdot)$. All restrictions of Sobolev functions on $\partial \Omega$ are understood in the sense of traces.

For every $r \in(1,+\infty)$, let $A_{r}: W^{1, r}(\Omega) \rightarrow W^{1, r}(\Omega)^{*}$ be defined by

$$
\left\langle A_{r}(u), h\right\rangle=\int_{\Omega}|D u|^{r-2}(D u, D h)_{\mathbb{R}^{N}} d z \text { for all } u, h \in W^{1, r}(\Omega) .
$$


The following proposition summarizes the main properties of this map (see Gasinski \& Papageorgiou [3]).

Proposition 2.1. The map $A_{r}(\cdot)$ is bounded (that is, maps bounded sets to bounded sets) continuous, monotone (hence maximal monotone, too) and of type $(S)_{+}$, that is, if $u_{n} \stackrel{w}{\rightarrow} u$ in $W^{1, r}(\Omega)$ and $\limsup \left\langle A_{r}\left(u_{n}\right), u_{n}-u\right\rangle$, then $u_{n} \rightarrow u$ in $W^{1, r}(\Omega)$.

Evidently, the $(S)_{+}$-property is useful in verifying the C-condition.

Now we introduce the conditions on the potential function $\xi(\cdot)$ and on the boundary coefficient $\beta(\cdot)$.

$H(\xi): \xi \in L^{\infty}(\Omega)$ and $\xi(z) \geqslant 0$ for almost all $z \in \Omega$.

$H(\beta): \beta \in C^{0, \alpha}(\partial \Omega)$ with $0<\alpha<1$ and $\beta(z) \geqslant 0$ for all $z \in \partial \Omega$.

$H_{0}: \xi \not \equiv 0$ or $\beta \not \equiv 0$.

Remark 2.1. When $\beta \equiv 0$ we have the usual Neumann problem.

The next two propositions can be found in Papageorgiou \& Rădulescu [10].

Proposition 2.2. If $\xi \in L^{\infty}(\Omega), \xi(z) \geqslant 0$ for almost all $z \in \Omega$ and $\xi \not \equiv 0$, then $c_{0}\|u\|^{p} \leqslant$ $\|D u\|_{p}^{p}+\int_{\Omega} \xi(z)|u|^{p} d z$ for some $c_{0}>0$ and all $u \in W^{1, p}(\Omega)$.

Proposition 2.3. If $\beta \in L^{\infty}(\partial \Omega), \beta(z) \geqslant 0$ for $\sigma$-almost all $z \in \partial \Omega$ and $\beta \not \equiv 0$, then $c_{1}\|u\|^{p} \leqslant$ $\|D u\|_{p}^{p}+\int_{\partial \Omega} \beta(z)|u|^{p} d \sigma$ for some $c_{1}>0$ and all $u \in W^{1, p}(\Omega)$.

In what follows, let $\gamma_{p}: W^{1, p}(\Omega) \rightarrow \mathbb{R}$ be defined by

$$
\gamma_{p}(u)=\|\left. D u\right|_{p} ^{p}+\int_{\Omega} \xi(z)|u|^{p} d z+\int_{\partial \Omega} \beta(z)|u|^{p} d \sigma \text { for all } u \in W^{1, p}(\Omega) .
$$

If hypotheses $H(\xi), H(\beta), H_{0}$ hold, then from Propositions 2.2 and 2.3 we can infer that

$$
c_{2}\|u\|^{p} \leqslant \gamma_{p}(u) \text { for some } c_{2}>0 \text { and all } u \in W^{1, p}(\Omega) .
$$

As we have already mentioned in the introduction, our approach involves also truncation and comparison techniques. So, the next strong comparison principle, a slight variant of Proposition 4 of Papageorgiou \& Smyrlis [17], will be useful.

Proposition 2.4. If $\hat{\xi} \in L^{\infty}(\Omega)$ with $\hat{\xi}(z) \geqslant 0$ for almost all $z \in \Omega, h_{1}, h_{2} \in L^{\infty}(\Omega)$,

$$
0<c_{3} \leqslant h_{2}(z)-h_{1}(z) \text { for almost all } z \in \Omega,
$$

and the functions $u_{1}, u_{2} \in C^{1}(\bar{\Omega}) \backslash\{0\}, u_{1} \leqslant u_{2}, u_{1}^{-\gamma}, u_{2}^{-\gamma} \in L^{\infty}(\Omega)$ satisfy

$$
\begin{aligned}
& -\Delta_{p} u_{1}-\Delta_{q} u_{1}+\hat{\xi}(z) u_{1}^{p-1}-u_{1}^{-\gamma}=h_{1} \text { for almost all } z \in \Omega, \\
& -\Delta_{p} u_{2}-\Delta_{q} u_{2}+\hat{\xi}(z) u_{2}^{p-1}-u_{2}^{-\gamma}=h_{2} \text { for almost all } z \in \Omega,
\end{aligned}
$$

then $u_{2}-u_{1} \in \operatorname{int} \hat{C}_{+}$.

Consider a Carathéodory function $f_{0}: \Omega \times \mathbb{R} \rightarrow \mathbb{R}$ satisfying

$$
\left|f_{0}(z, x)\right| \leqslant a_{0}(z)\left[1+|x|^{r-1}\right] \text { for almost all } z \in \Omega \text { and all } x \in \mathbb{R}
$$

with $a_{0} \in L^{\infty}(\Omega)$ and $1<r \leqslant p^{*}=\left\{\begin{array}{ll}\frac{N p}{N-p} & \text { if } p<N \\ +\infty & \text { if } N \leqslant p\end{array}\right.$ (the critical Sobolev exponent corresponding to $p$ ).

We set $F_{0}(z, x)=\int_{0}^{x} f_{0}(z, s) d s$ and consider the $C^{1}$-functional $\varphi_{0}: W^{1, p}(\Omega) \rightarrow \mathbb{R}$ defined by

$$
\left.\varphi_{0}(u)=\frac{1}{p} \gamma_{p}(u)+\frac{1}{q}\|D u\|_{q}^{q}-\int_{\Omega} F_{0}(z, u) d z \text { for all } u \in W^{1, p}(\Omega) \text { (recall that } q<p\right) .
$$

The next proposition can be found in Papageorgiou \& Rădulescu [9] and essentially is an outgrowth of the nonlinear regularity theory of Lieberman [7]. 
Proposition 2.5. If $u_{0} \in W^{1, p}(\Omega)$ is a local $C^{1}(\bar{\Omega})$-minimizer of $\varphi_{0}$, that is, there exists $\rho_{0}>0$ such that

$$
\varphi_{0}\left(u_{0}\right) \leqslant \varphi_{0}\left(u_{0}+h\right) \text { for all }\|h\|_{C^{1}(\bar{\Omega})} \leqslant \rho_{0}
$$

then $u_{0} \in C^{1, \alpha}(\bar{\Omega})$ for some $\alpha \in(0,1)$ and $u_{0}$ is also a local $W^{1, p}(\Omega)$-minimizer of $\varphi_{0}$, that is, there exists $\rho_{1}>0$ such that

$$
\varphi_{0}\left(u_{0}\right) \leqslant \varphi_{0}(u+h) \text { for all }\|h\| \leqslant \rho_{1} .
$$

The next fact about ordered Banach spaces is useful in producing upper bounds for functions and can be found in Gasinski \& Papageorgiou [4, Problem 4.180, p. 680].

Proposition 2.6. If $X$ is an ordered Banach space with positive (order) cone $K$,

$$
\text { int } K \neq \emptyset \text { and } e \in \operatorname{int} K
$$

then for every $u \in X$ we can find $\lambda_{u}>0$ such that $\lambda_{u} e-u \in K$.

Under hypotheses $H(\xi), H(\beta), H_{0}$, the differential operator $u \mapsto-\Delta_{p} u+\xi(z)|u|^{p-2} u$ with the Robin boundary condition, has a principal eigenvalue $\hat{\lambda}_{1}(p)>0$ which is isolated, simple and admits the following variational characterization:

$$
\hat{\lambda}_{1}(p)=\inf \left\{\frac{\gamma_{p}(u)}{\|u\|_{p}^{p}}: u \in W^{1, p}(\Omega), u \neq 0\right\} .
$$

The infimum is realized on the corresponding one-dimensional eigenspace, the elements of which have fixed sign. By $\hat{u}_{1}(p)$ we denote the positive, $L^{p}$-normalized (that is, $\left\|\hat{u}_{1}(p)\right\|_{p}=1$ ) eigenfunction corresponding to $\hat{\lambda}_{1}(p)>0$. The nonlinear Hopf theorem (see, for example, Gasinski \& Papageorgiou $[3$, p. 738$])$ implies that $\hat{u}_{1}(p) \in D_{+}$.

Let us fix some basic notation which we will use throughout this work. So, if $x \in \mathbb{R}$, we set $x^{ \pm}=\max \{ \pm x, 0\}$ and the for $u \in W^{1, p}(\Omega)$ we define $u^{ \pm}(z)=u(z)^{ \pm}$for all $z \in \Omega$. We know that

$$
u^{ \pm} \in W^{1, p}(\Omega), u=u^{+}-u^{-},|u|=u^{+}+u^{-} .
$$

If $\varphi \in C^{1}\left(W^{1, p}(\Omega), \mathbb{R}\right)$, then by $K_{\varphi}$ we denote the critical set of $\varphi$, that is,

$$
K_{\varphi}=\left\{u \in W^{1, p}(\Omega): \varphi^{\prime}(u)=0\right\} .
$$

Also, if $u, y \in W^{1, p}(\Omega)$, with $u \leqslant y$, then we define

$$
\begin{aligned}
& {[u, y]=\left\{h \in W^{1, p}(\Omega): u(z) \leqslant h(z) \leqslant y(z) \text { for almost all } z \in \Omega\right\},} \\
& {[u)=\left\{h \in W^{1, p}(\Omega): u(z) \leqslant h(z) \text { for almost all } z \in \Omega\right\},} \\
& \operatorname{int}_{C^{1}(\bar{\Omega})}[u, y]=\text { the interior in the } C^{1}(\bar{\Omega}) \text {-norm of }[u, y] \cap C^{1}(\bar{\Omega}) .
\end{aligned}
$$

Now we introduce our hypotheses on the perturbation $f(z, x)$.

$H(f): f: \Omega \times \mathbb{R} \rightarrow \mathbb{R}$ is a Carathéodory function such that $f(z, 0)=0$ for almost all $z \in \Omega$ and

(i) $f(z, x) \leqslant a(z)\left(1+x^{r-1}\right)$ for almost all $z \in \Omega$ and all $x \geqslant 0$ with $a \in L^{\infty}(\Omega), p<r<p^{*}$;

(ii) if $F(z, x)=\int_{0}^{x} f(z, s) d s$, then $\lim _{x \rightarrow+\infty} \frac{F(z, x)}{x^{p}}=+\infty$ uniformly for almost all $z \in \Omega$;

(iii) there exists $\tau \in\left((r-p) \max \left\{\frac{N}{p}, 1\right\}, p^{*}\right)$ such that

$$
0<\hat{\beta}_{0} \leqslant \liminf _{x \rightarrow+\infty} \frac{f(z, x) x-p F(z, x)}{x^{\tau}} \text { uniformly for almost all } z \in \Omega ;
$$

(iv) for every $\vartheta>0$, there exists $m_{\vartheta}>0$ such that

$$
m_{\vartheta} \leqslant f(z, x) \text { for almost all } z \in \Omega \text { and all } x \geqslant \vartheta
$$

(v) for every $\rho>0$ and $\lambda>0$, there exists $\hat{\xi}_{\rho}^{\lambda}>0$ such that for almost all $z \in \Omega$, the function $x \mapsto f(z, x)+\hat{\xi}_{\rho}^{\lambda} x^{p-1}$ is nondecreasing on $[0, \rho]$.

Remark 2.2. Since we are looking for positive solutions and the above hypotheses concern the positive semiaxis, without any loss of generality we may assume that

$$
f(z, x)=0 \text { for almost all } z \in \Omega \text { and all } x \leqslant 0 .
$$


From hypotheses $H(f),($ ii $),($ iii $)$ it follows that

$$
\lim _{x \rightarrow+\infty} \frac{f(z, x)}{x^{p-1}}=+\infty \text { uniformly for almost all } z \in \Omega .
$$

Hence, for almost all $z \in \Omega$ the perturbation $f(z, \cdot)$ is $(p-1)$-superlinear near $+\infty$. However, this superlinearity of $f(z, \cdot)$ is not expressed using the well-known AR-condition. We recall that the AR-condition (unilateral version due to (3)) says that there exist $q>p$ and $M>0$ such that

$$
\begin{aligned}
& 0<q F(z, x) \leqslant f(z, x) x \text { for almost all } z \in \Omega \text { and all } x \geqslant M, \\
& 0<\underset{\Omega}{\operatorname{essinf} F(\cdot, M) .}
\end{aligned}
$$

Integrating (3a) and using (3b), we obtain the weaker condition

$$
\begin{aligned}
& c_{4} x^{q} \leqslant F(z, x) \text { for almost all } z \in \Omega \text { all } x \geqslant M, \text { and some } c_{4}>0, \\
\Rightarrow & c_{4} x^{q-1} \leqslant f(z, x) \text { for almost all } z \in \Omega \text { and all } x \geqslant M .
\end{aligned}
$$

So, the AR-condition dictates an at least $(q-1)$-polynomial growth for $f(z, \cdot)$. Here we replace the AR-condition with hypothesis $H(f)($ iii $)$ which is less restrictive and permits superlinear nonlinearities with "slower" growth near $+\infty$. For example the function

$$
f(x)=x^{p-1} \ln (1+x) \text { for all } x \geqslant 0 .
$$

(for the sake of simplicity we have dropped the $z$-dependence) satisfies hypotheses $H(f)$, but fails to satisfy the AR-condition.

We introduce the following sets:

$$
\begin{aligned}
& \mathcal{L}=\left\{\lambda>0: \text { problem }\left(P_{\lambda}\right) \text { has a positive solution }\right\} \\
& S_{\lambda}=\text { the set of positive solutions of }\left(P_{\lambda}\right) .
\end{aligned}
$$

Also we set

$$
\lambda^{*}=\sup \mathcal{L}
$$

\section{Some AuXiliary Robin PRoblems}

Let $\eta>0$. First we examine the following auxiliary Robin problem

$$
\left\{\begin{array}{l}
-\Delta_{p} u(z)-\Delta_{q} u(z)+\xi(z) u(z)^{p-1}=\eta \text { in } \Omega, \\
\frac{\partial u}{\partial n_{p q}}+\beta(z) u^{p-1}=0 \text { on } \partial \Omega, u>0 .
\end{array}\right\}
$$

Proposition 3.1. If hypotheses $H(\xi), H(\beta), H_{0}$ hold, then for every $\eta>0$ problem (5) has a unique solution $\tilde{u}_{\eta} \in D_{+}$, the mapping $\eta \mapsto \tilde{u}_{\eta}$ is strictly increasing (that is, $\eta<\eta^{\prime} \Rightarrow \tilde{u}_{\eta^{\prime}}-\tilde{u}_{\eta} \in \operatorname{int} \hat{C}_{+}$) and

$$
\tilde{u}_{\eta} \rightarrow 0 \text { in } C^{1}(\bar{\Omega}) \text { as } \eta \rightarrow 0^{+} \text {. }
$$

Proof. Consider the map $V: W^{1, p}(\Omega) \rightarrow W^{1, p}(\Omega)^{*}$ defined by

$$
\begin{array}{r}
\langle V(u), h\rangle=\left\langle A_{p}(u), h\right\rangle+\left\langle A_{q}(u), h\right\rangle+\int_{\Omega} \xi(z)|u|^{p-2} u h d z+\int_{\partial \Omega} \beta(z)|u|^{p-2} u h d \sigma \\
\text { for all } u, h \in W^{1, p}(\Omega) .
\end{array}
$$

Evidently, $V(\cdot)$ is continuous, strictly monotone (hence maximal monotone, too) and coercive (see (1)). Therefore $V(\cdot)$ is surjective (see Gasinski \& Papageorgiou [3, Corollary 3.2.31, p. 319]). So, we can find $\tilde{u}_{\eta} \in W^{1, p}(\Omega), \tilde{u}_{\eta} \neq 0$ such that

$$
V\left(\tilde{u}_{\eta}\right)=\eta \text {. }
$$

The strict monotonicity of $V(\cdot)$ implies that $\tilde{u}_{\eta}$ is unique. We have

$$
\left\langle V\left(\tilde{u}_{\eta}\right), h\right\rangle=\eta \int_{\Omega} h d z \text { for all } h \in W^{1, p}(\Omega) .
$$


In $(7)$ we choose $h=-\tilde{u}_{\eta}^{-} \in W^{1, p}(\Omega)$. Then

$$
\begin{aligned}
& c_{2}\left\|\tilde{u}_{\eta}^{-}\right\|^{p} \leqslant 0(\text { see }(1)), \\
\Rightarrow \quad & \tilde{u}_{\eta} \geqslant 0, \tilde{u}_{\eta} \neq 0 .
\end{aligned}
$$

From (7) we have

$$
\left\{\begin{array}{l}
-\Delta_{p} \tilde{u}_{\eta}(z)-\Delta_{q} \tilde{u}_{\eta}(z)+\xi(z) \tilde{u}_{\eta}(z)^{p-1}=\eta \text { for almost all } z \in \Omega, \\
\frac{\partial \tilde{u}_{\eta}}{\partial n_{p q}}+\beta(z) \tilde{u}_{\eta}^{p-1}=0 \text { on } \partial \Omega .
\end{array}\right\}
$$

From (8) and Proposition 7 of Papageorgiou \& Rădulescu [9] we deduce that

$$
\tilde{u}_{\eta} \in L^{\infty}(\Omega) \text {. }
$$

Then the nonlinear regularity theory of Lieberman [7] implies that

$$
\tilde{u}_{\eta} \in C_{+} \backslash\{0\} \text {. }
$$

From (8) we have

$$
\begin{aligned}
& \Delta_{p} \tilde{u}_{\eta}(z)+\Delta_{q} \tilde{u}_{\eta}(z) \leqslant\|\xi\|_{\infty} \tilde{u}_{\eta}(z)^{p-1} \text { for almost all } z \in \Omega, \\
\Rightarrow \quad & \tilde{u}_{\eta} \in D_{+}(\text {see Pucci \& Serrin }[21, \text { pp. 111, 120]). }
\end{aligned}
$$

Suppose that $0<\eta_{1}<\eta_{2}$ and let $\tilde{u}_{\eta_{1}}, \tilde{u}_{\eta_{2}} \in D_{+}$be the corresponding solutions of problem (5). We have

$$
\begin{aligned}
& -\Delta_{p} \tilde{u}_{\eta_{1}}-\Delta_{q} \tilde{u}_{\eta_{1}}+\xi(z) \tilde{u}_{\eta_{1}}^{p-1}=\eta_{1}<\eta_{2}=-\Delta_{p} \tilde{u}_{\eta_{2}}-\Delta_{q} \tilde{u}_{\eta_{2}}+\xi(z) \tilde{u}_{\eta_{2}} \\
& \text { for almost all } z \in \Omega, \\
\Rightarrow \quad & \left.\tilde{u}_{\eta_{2}}-\tilde{u}_{\eta_{1}} \in \text { int } \hat{C}_{+} \text {(see Proposition } 2.4\right), \\
\Rightarrow & \eta \mapsto \tilde{u}_{\eta} \text { is strictly increasing from }(0,+\infty) \text { into } C^{1}(\bar{\Omega}) .
\end{aligned}
$$

Finally, let $\eta_{n} \rightarrow 0^{+}$and let $\tilde{u}_{n}=\tilde{u}_{\eta_{n}} \in D_{+}$be the corresponding solutions of (5). As before, via Proposition 7 of Papageorgiou \& Rădulescu [9], we can find $c_{5}>0$ such that

$$
\left\|\tilde{u}_{n}\right\|_{\infty} \leqslant c_{5} \text { for all } n \in \mathbb{N} \text {. }
$$

Then from Lieberman [7] we infer that there exist $\alpha \in(0,1)$ and $c_{6}>0$ such that

$$
\tilde{u}_{n} \in C^{1, \alpha}(\bar{\Omega}),\left\|\tilde{u}_{n}\right\|_{C^{1, \alpha}(\bar{\Omega})} \leqslant c_{6} \text { for all } n \in \mathbb{N} .
$$

Exploiting the compact embedding of $C^{1, \alpha}(\bar{\Omega})$ into $C^{1}(\bar{\Omega})$, the monotonicity of the sequence $\left\{\tilde{u}_{n}\right\}_{n \geqslant 1} \subseteq D_{+}$and that for $\eta=0, u \equiv 0$ is the only solution of (5) we obtain

$$
\tilde{u}_{n} \rightarrow 0 \text { in } C^{1}(\bar{\Omega}) .
$$

The proof is now complete.

Using Proposition 3.1, we see that we can find $\eta_{0}>0$ such that

$$
\eta \leqslant \tilde{u}_{\eta}(z)^{-\gamma} \text { for all } z \in \bar{\Omega}, \quad 0<\eta \leqslant \eta_{0} .
$$

We consider the following purely singular problem

$$
\left\{\begin{array}{c}
-\Delta_{p} u(z)-\Delta_{q} u(z)+\xi(z) u(z)^{p-1}=u(z)^{-\gamma} \text { in } \Omega \\
\frac{\partial u}{\partial n_{p q}}+\beta(z) u^{p-1}=0 \text { on } \partial \Omega, u>0,0<\gamma<1 .
\end{array}\right\}
$$

In the first place, by a solution of (10) we understand a weak solution, that is, a function $u \in$ $W^{1, p}(\Omega)$ such that

$$
\begin{aligned}
& u^{-\gamma} h \in L^{1}(\Omega) \text { and }\left\langle A_{p}(u), h\right\rangle+\left\langle A_{q}(u), h\right\rangle+\int_{\Omega} \xi(z) u^{p-1} h d z+\int_{\partial \Omega} \beta(z) u^{p-1} h d \sigma \\
& =\int_{\Omega} u^{-\gamma} h d z \text { for all } h \in W^{1, p}(\Omega) .
\end{aligned}
$$

In fact, using the nonlinear regularity theory, we will be able to establish more regularity for the solution of (10), which in fact, is a strong solution (that is, the equation can be interpreted pointwise almost everywhere on $\Omega$ ). 
Proposition 3.2. If hypotheses $H(\xi), H(\beta), H_{0}$ hold, then problem (10) admits a unique solution $v \in D_{+}$.

Proof. Let $\eta \in\left(0, \eta_{0}\right]$ (see $\left.(9)\right)$ and recall that $\tilde{u}_{\eta} \in D_{+}$. So $m_{\eta}=\min _{\bar{\Omega}} \tilde{u}_{\eta}>0$ and

$$
\begin{aligned}
& \eta \leqslant \tilde{u}_{\eta}^{-\gamma} \leqslant m_{\eta}^{-\gamma}(\text { see }(9)), \\
\Rightarrow \quad & \tilde{u}_{\eta}^{-\gamma} \in L^{\infty}(\Omega) .
\end{aligned}
$$

We consider the following truncation of the reaction in problem (10):

$$
k(z, x)= \begin{cases}\tilde{u}_{\eta}(z)^{-\gamma} & \text { if } x \leqslant \tilde{u}_{\eta}(z) \\ x^{-\gamma} & \text { if } \tilde{u}_{\eta}(z)<x\end{cases}
$$

This is a Carathéodory function. We set $K(z, x)=\int_{0}^{x} k(z, s) d s$ and consider the $C^{1}$-functional $\Psi: W^{1, p}(\Omega) \rightarrow \mathbb{R}$ defined by

$$
\Psi(u)=\frac{1}{p} \gamma_{p}(u)+\frac{1}{q}\|D u\|_{q}^{q}-\int_{\Omega} K(z, u) d z \text { for all } u \in W^{1, p}(\Omega) .
$$

From (12) and (11), we see that $\Psi(\cdot)$ is coercive. Also the Sobolev embedding theorem and the compactness of the trace map, imply that $\Psi(\cdot)$ is sequentially weakly lower semicontinuous. So, we can find $v \in W^{1, p}(\Omega)$ such that

$$
\begin{array}{cc} 
& \Psi(v)=\inf \left\{\Psi(u): u \in W^{1, p}(\Omega)\right\}, \\
& \Psi^{\prime}(v)=0, \\
\Rightarrow & \left\langle A_{p}(v), h\right\rangle+\left\langle A_{q}(v), h\right\rangle+\int_{\Omega} \xi(z)|v|^{p-2} v h d z+\int_{\partial \Omega} \beta(z)|v|^{p-2} v h d \sigma= \\
& \int_{\Omega} k(z, v) h d z \text { for all } h \in W^{1, p}(\Omega) .
\end{array}
$$

In (13) we choose $\left(\tilde{u}_{\eta}-v\right)^{+} \in W^{1, p}(\Omega)$. Then

$$
\begin{aligned}
& \left\langle A_{p}(v),\left(\tilde{u}_{\eta}-v\right)^{+}\right\rangle+\left\langle A_{q}(v),\left(\tilde{u}_{\eta}-v\right)^{+}\right\rangle+\int_{\Omega} \xi(z)|v|^{p-2} v\left(\tilde{u}_{\eta}-v\right)^{+} d z+ \\
& \int_{\partial \Omega} \beta(z)|v|^{p-2} v\left(\tilde{u}_{\eta}-v\right)^{+} d \sigma=\int_{\Omega} \tilde{u}_{\eta}^{-\gamma}\left(\tilde{u}_{\eta}-v\right)^{+} d z(\text { see }(12)) \\
\geqslant & \left.\int_{\Omega} \eta\left(\tilde{u}_{\eta}-v\right)^{+} d z \text { (see }(9) \text { and recall that } 0<\eta \leqslant \eta_{0}\right) \\
= & \left\langle A_{p}\left(\tilde{u}_{\eta}\right),\left(\tilde{u}_{\eta}-v\right)^{+}\right\rangle+\left\langle A_{q}\left(\tilde{u}_{\eta}\right),\left(\tilde{u}_{\eta}-v\right)^{+}\right\rangle+\int_{\Omega} \xi(z) \tilde{u}_{\eta}^{p-1}\left(\tilde{u}_{\eta}-v\right)^{+} d z+ \\
& \int_{\partial \Omega} \beta(z) \tilde{u}_{\eta}^{p-1}\left(\tilde{u}_{\eta}-v\right)^{+} d \sigma \text { (see Proposition 3.1), } \\
\Rightarrow & \tilde{u}_{\eta} \leqslant v .
\end{aligned}
$$

Then from (12), (13), (14) we obtain

$$
\left\{\begin{array}{l}
-\Delta_{p} v(z)-\Delta_{q} v(z)+\xi(z) v(z)^{p-1}=v(z)^{-\gamma} \text { for almost all } z \in \Omega, \\
\frac{\partial v}{\partial n_{p q}}+\beta(z) v^{p-1}=0 \text { on } \partial \Omega
\end{array}\right\}
$$

(see Papageorgiou \& Rădulescu [8]).

From (14) we have $v^{-\gamma} \leqslant \tilde{u}_{\eta}^{-\gamma} \in L^{\infty}(\Omega)$ (see (11)). So, from (15) and [9] we have $v \in L^{\infty}(\Omega)$. Then the nonlinear regularity theory of Lieberman [7] implies that $v \in C_{+}$. Hence it follows from (14) that

$$
v \in D_{+}
$$


Next, we show that this positive solution is unique. To this end, let $\hat{v} \in W^{1, p}(\Omega)$ be another positive solution of (10). Again we have $\hat{v} \in D_{+}$. Then

$$
\begin{aligned}
& \left\langle A_{p}(v),(\hat{v}-v)^{+}\right\rangle+\left\langle A_{q}(v),(\hat{v}-v)^{+}\right\rangle+\int_{\Omega} \xi(z) v^{p-1}(\hat{v}-v)^{+} d z+ \\
& \int_{\partial \Omega} \beta(z) v^{p-1}(\hat{v}-v)^{+} d \sigma \\
= & \int_{\Omega} v^{-\gamma}(\hat{v}-v)^{+} d z \\
\geqslant & \int_{\Omega} \hat{v}^{-\gamma}(\hat{v}-v)^{+} d z \\
= & \left\langle A_{p}(\hat{v}),(\hat{v}-v)^{+}\right\rangle+\left\langle A_{q}(\hat{v}),(\hat{v}-v)^{+}\right\rangle+\int_{\Omega} \xi(z) \hat{v}^{p-1}(\hat{v}-v)^{+} d z+ \\
& \int_{\partial \Omega} \beta(z) \hat{v}^{p-1}(\hat{v}-v)^{+} d \sigma \\
\Rightarrow & \hat{v} \leqslant v .
\end{aligned}
$$

Interchanging the roles of $v$ and $\hat{v}$ in the above argument, we obtain

$$
\begin{aligned}
v & \leqslant \hat{v}, \\
\Rightarrow \quad v & =\hat{v} .
\end{aligned}
$$

This proves the uniqueness of the positive solution of the purely singular problem (10).

Next, we consider the following nonlinear Robin problem

$$
\left\{\begin{array}{l}
-\Delta_{p} u(z)-\Delta_{q} u(z)+\xi(z) u(z)^{p-1}=v(z)^{-\gamma}+1 \text { in } \Omega, \\
\frac{\partial u}{\partial n_{p q}}+\beta(z) u^{p-1}=0 \text { on } \partial \Omega, u>0 .
\end{array}\right\}
$$

Proposition 3.3. If hypotheses $H(\xi), H(\beta), H_{0}$ hold, then problem (16) admits a unique solution $\bar{u} \in D_{+}$and $v \leqslant \bar{u}$.

Proof. We know that $v^{-\gamma} \in L^{\infty}(\Omega)$ (see (11) and (14)). Then the existence and uniqueness of the solution $\bar{u} \in W^{1, p}(\Omega) \backslash\{0\}, \bar{u} \geqslant 0$ of (16) follow from the surjectivity and strict monotonicity of the map $V(\cdot)$ (see the proof of Proposition 3.1). The nonlinear regularity theory and the nonlinear Hopf's theorem imply that $\bar{u} \in D_{+}$.

Moreover, we have

$$
\begin{aligned}
& \left\langle A_{p}(\bar{u}),(v-\bar{u})^{+}\right\rangle+\left\langle A_{q}(\bar{u}),(v-\bar{u})^{+}\right\rangle+\int_{\Omega} \xi(z) \bar{u}^{p-1}(v-\bar{u})^{+} d z+ \\
& \int_{\partial \Omega} \beta(z) \bar{u}^{p-1}(v-\bar{u})^{+} d \sigma \\
= & \int_{\Omega}\left[v^{-\gamma}+1\right](v-\bar{u})^{+} d z(\text { see }(16)) \\
\geqslant & \int_{\Omega} v^{-\gamma}(v-\bar{u})^{+} d z \\
= & \left\langle A_{p}(v),(v-\bar{u})^{+}\right\rangle+\left\langle A_{q}\left(v,(v-\bar{v})^{+}\right)\right\rangle+\int_{\Omega} \xi(z) v^{p-1}(v-\bar{v})^{+} d z+ \\
& \int_{\partial \Omega} \beta(z) v^{p-1}(v-\bar{v})^{+} d \sigma \\
\Rightarrow & v \leqslant \bar{u} .
\end{aligned}
$$

The proof is now complete. 


\section{Positive solutions}

In this section we prove the bifurcation-type theorem described in the Introduction.

Proposition 4.1. If hypotheses $H(\xi), H(\beta), H_{0}, H(f)$ hold, then $\mathcal{L} \neq \emptyset$ and $S_{\lambda} \subseteq D_{+}$.

Proof. Let $v \in D_{+}$be the unique positive solution of the auxiliary problem (10) (see Proposition 3.2) and $\bar{u} \in D_{+}$the unique solution of (16) (see Proposition 3.3). We know that $v \leqslant \bar{u}$ (see Proposition 3.3). Since $\bar{u} \in D_{+}$, hypothesis $H(f)(i)$ implies that

$$
0 \leqslant f(z, \bar{u}(z)) \leqslant c_{7} \text { for some } c_{7}>0 \text { and almost all } z \in \Omega .
$$

So, we can find $\lambda_{0}>0$ small such that

$$
0 \leqslant \lambda f(z, \bar{u}(z)) \leqslant 1 \text { for almost all } z \in \Omega \text { and all } 0<\lambda \leqslant \lambda_{0} .
$$

We consider the following truncation of the reaction in problem $\left(P_{\lambda}\right)$

$$
\vartheta_{\lambda}(z, x)= \begin{cases}v(z)^{-\gamma}+\lambda f(z, v(z)) & \text { if } x<v(z) \\ x^{-\gamma}+\lambda f(z, x) & \text { if } v(z) \leqslant x \leqslant \bar{u}(z) \\ \bar{u}(z)^{-\gamma}+\lambda f(z, \bar{u}(z)) & \text { if } \bar{u}(z)<x\end{cases}
$$

This is a Carathéodory function. We set $\theta_{\lambda}(z, x)=\int_{0}^{x} \vartheta_{\lambda}(z, s) d s$ and consider the functional $\mu_{\lambda}: W^{1, p}(\Omega) \rightarrow \mathbb{R}\left(\lambda \in\left(0, \lambda_{0}\right]\right)$ defined by

$$
\mu_{\lambda}(u)=\frac{1}{p} \gamma_{p}(u)+\frac{1}{q}\|D u\|_{q}^{q}-\int_{\Omega} \theta_{\lambda}(z, u) d z \text { for all } u \in W^{1, p}(\Omega)
$$

Since $0 \leqslant \bar{u}^{-\gamma} \leqslant v^{-\gamma} \in L^{\infty}(\Omega)$, we see that $\mu_{\lambda} \in C^{1}\left(W^{1, p}(\Omega)\right)$. Also, it is clear from (18) and $(1)$, that $\mu_{\lambda}(\cdot)$ is coercive. In addition, it is sequentially weakly lower semicontinuous. So, we can find $u_{\lambda} \in W^{1, p}(\Omega)$ such that

$$
\begin{aligned}
& \mu_{\lambda}\left(u_{\lambda}\right)=\inf \left\{\mu_{\lambda}(u): u \in W^{1, p}(\Omega)\right\} \\
& \Rightarrow \mu_{\lambda}^{\prime}\left(u_{\lambda}\right)=0 \\
\Rightarrow & \left\langle A_{p}\left(u_{\lambda}\right), h\right\rangle+\left\langle A_{q}\left(u_{\lambda}\right), h\right\rangle+\int_{\Omega} \xi(z)\left|u_{\lambda}\right|^{p-2} u_{\lambda} h d z+\int_{\partial \Omega} \beta(z)\left|u_{\lambda}\right|^{p-2} u_{\lambda} h d \sigma \\
= & \int_{\Omega} \vartheta_{\lambda}\left(z, u_{\lambda}\right) h d z \text { for all } h \in W^{1, p}(\Omega) .
\end{aligned}
$$

In (19) first we choose $h=\left(u_{\lambda}-\bar{u}\right)^{+} \in W^{1, p}(\Omega)$. Then

$$
\begin{aligned}
& \left\langle A_{p}\left(u_{\lambda}\right),\left(u_{\lambda}-\bar{u}\right)^{+}\right\rangle+\left\langle A_{q}\left(u_{\lambda}\right),\left(u_{\lambda}-\bar{u}\right)^{+}\right\rangle+\int_{\Omega} \xi(z) u_{\lambda}^{p+}\left(u_{\lambda}-\bar{u}\right)^{+} d z+ \\
& \int_{\partial \Omega} \beta(z) u_{\lambda}^{p-1}\left(u_{\lambda}-\bar{u}\right) d \sigma \\
& \left.=\int_{\Omega}\left[\bar{u}^{-\gamma}+\lambda f(z, \bar{u})\right]\left(u_{\lambda}-\bar{u}\right)^{+} d z(\text { see }(18))\right) \\
& \leqslant \int_{\Omega}\left[\bar{u}^{-\gamma}+1\right]\left(u_{\lambda}-\bar{u}\right)^{+} d z(\text { see }(17)) \\
& \leqslant \int_{\Omega}\left[v^{-\gamma}+1\right]\left(u_{\lambda}-\bar{u}\right)^{+} d z(\text { since } v \leqslant \bar{u}) \\
& =\left\langle A_{p}(\bar{u}),\left(u_{\lambda}-\bar{u}\right)^{+}\right\rangle+\left\langle A_{q}(\bar{u}),\left(u_{\lambda}-\bar{u}\right)^{+}\right\rangle+\int_{\Omega} \xi(z) \bar{u}^{p-1}\left(u_{\lambda}-\bar{u}\right)^{+} d z \\
& +\int_{\partial \Omega} \beta(z) \bar{u}^{p-1}\left(u_{\lambda}-\bar{u}\right)^{+} d \sigma \text { (see Proposition 3.3), } \\
& \Rightarrow u_{\lambda} \leqslant \bar{u} .
\end{aligned}
$$


Next, in (19) we choose $h=\left(v-u_{\lambda}\right)^{+} \in W^{1, p}(\Omega)$. Then

$$
\begin{aligned}
& \left\langle A_{p}\left(u_{\lambda}\right),\left(v-u_{\lambda}\right)^{+}\right\rangle+\left\langle A_{q}\left(u_{\lambda}\right),\left(v-u_{\lambda}\right)^{+}\right\rangle+\int_{\Omega} \xi(z)\left|u_{\lambda}\right|^{p-2} u_{\lambda}\left(v-u_{\lambda}\right)^{+} d z+ \\
& \int_{\partial \Omega} \beta(z)\left|u_{\lambda}\right|^{p-2} u_{\lambda}\left(v-u_{\lambda}\right)^{+} d \sigma \\
& =\int_{\Omega}\left[v^{-\gamma}+\lambda f(z, v)\right]\left(v-u_{\lambda}\right)^{+} d z(\text { see }(18)) \\
& \geqslant \int_{\Omega} v^{-\gamma}\left(v-u_{\lambda}\right)^{+} d z(\text { since } f \geqslant 0) \\
& =\left\langle A_{p}(v),\left(v-u_{\lambda}\right)^{+}\right\rangle+\left\langle A_{q}(v),\left(v-u_{\lambda}\right)^{+}\right\rangle+\int_{\lambda} \xi(z) v^{p-1}\left(v-u_{\lambda}\right)^{+} d z \\
& +\int_{\partial \Omega} \beta(z) v^{p-1}\left(v-u_{\lambda}\right)^{+} d \sigma(\text { see Proposition 3.2), } \\
& \Rightarrow v \leqslant u_{\lambda} .
\end{aligned}
$$

So, we have proved that

$$
u_{\lambda} \in[v, \bar{u}]
$$

From (18), (19), (20) it follows that

$$
\left\{\begin{array}{l}
-\Delta_{p} u_{\lambda}(z)-\Delta_{q} u_{\lambda}(z)+\xi(z) u_{\lambda}(z)^{p-1}=u_{\lambda}(z)^{-\gamma}+\lambda f\left(z, u_{\lambda}(z)\right) \\
\text { for almost all } z \in \Omega, \\
\frac{\partial u_{\lambda}}{\partial n_{p q}}+\beta(z) u_{\lambda}^{p-1}=0 \text { on } \partial \Omega,(\text { see }[8]) .
\end{array}\right\}
$$

From (21) and Proposition 3.1 of Papageorgiou \& Rădulescu [9], we have that $u_{\lambda} \in L^{\infty}(\Omega)$. So, the nonlinear regularity theory of Lieberman [7] implies that $u_{\lambda} \in D_{+}($see $(20))$. Therefore we have proved that

$$
\left(0, \lambda_{0}\right] \leqslant \mathcal{L} \neq \emptyset \text { and } S_{\lambda} \subseteq D_{+}
$$

The proof is now complete.

Next, we establish a lower bound for the elements of $S_{\lambda}$.

Proposition 4.2. If hypotheses $H(\xi), H(\beta), H_{0}, H(f)$ hold, $\lambda \in \mathcal{L}$ and $u \in S_{\lambda}$, then $v \leqslant u$.

Proof. From Proposition 4.1 we know that $u \in D_{+}$. Then Proposition 3.1 implies that for $\eta>0$ small we have $\tilde{u}_{\eta} \leqslant u$. So, we can define the following Carathéodory function

$$
e(z, x)= \begin{cases}\tilde{u}_{\eta}(z)^{-\gamma} & \text { if } x<\tilde{u}_{\eta}(z) \\ x^{-\gamma} & \text { if } \tilde{u}_{\eta}(z) \leqslant x \leqslant u(z) \\ u(z)^{-\gamma} & \text { if } u(z)<x\end{cases}
$$

We set $E(z, x)=\int_{0}^{x} e(z, s) d s$ and consider the functional $d: W^{1, p}(\Omega) \rightarrow \mathbb{R}$ defined by

$$
d(u)=\frac{1}{p} \gamma_{p}(u)+\frac{1}{q}\|D u\|_{q}^{q}-\int_{\Omega} E(z, u) d z \text { for all } u \in W^{1, p}(\Omega) .
$$

As before, we have $d \in C^{1}\left(W^{1, p}(\Omega)\right)$. Also, $d(\cdot)$ is coercive (see $\left.(22)\right)$ and weakly lower semicontinuous. Hence we can find $\hat{v} \in W^{1, p}(\Omega)$ such that

$$
\begin{aligned}
& d(\hat{u})=\inf \left\{d(u): u \in W^{1, p}(\Omega)\right\}, \\
\Rightarrow \quad & d^{\prime}(\hat{v})=0, \\
\Rightarrow \quad & \left\langle A_{p}(\hat{v}), h\right\rangle+\left\langle A_{q}(\hat{v}), h\right\rangle+\int_{\Omega} \xi(z)|\hat{v}|^{p-2} \hat{v} h d z+\int_{\partial \Omega} \beta(z)|\hat{v}|^{p-2} \hat{v} h d \sigma= \\
& \int_{\Omega} e(z, \hat{v}) h d z \text { for all } h \in W_{1, p}(\Omega) .
\end{aligned}
$$


In (23) first we choose $h=(\hat{v}-u)^{+} \in W^{1, p}(\Omega)$. Exploiting the fact that $u \in S_{\lambda}$ and recalling that $f \geqslant 0$, we obtain $\hat{v} \leqslant u$. Next in $(23)$ we test with $h=\left(\tilde{u}_{\eta}-v\right)^{+} \in W^{1, p}(\Omega)$. Using (22), (9) and Proposition 3.1, we obtain $\tilde{u}_{\eta} \leqslant \hat{v}$. Therefore

$$
\hat{v} \in\left[\tilde{u}_{\eta}, u\right] .
$$

From (22), (23), (24) and Proposition 3.2, we conclude that

$$
\begin{aligned}
& \hat{v}=v, \\
\Rightarrow \quad & v \leqslant u \text { for all } u \in S_{\lambda} .
\end{aligned}
$$

The proof is now complete.

Now we can deduce a structural property of $\mathcal{L}$.

Proposition 4.3. If hypotheses $H(\xi), H(\beta), H_{0}, H(f)$ hold, $\lambda \in \mathcal{L}, 0<\mu<\lambda$ and $u_{\lambda} \in S_{\lambda} \subseteq D_{+}$, then $\mu \in \mathcal{L}$ and we can find $u_{\mu} \in S_{\mu} \subseteq D_{+}$such that $u_{\lambda}-u_{\mu} \in \operatorname{int} \hat{C}_{+}$.

Proof. From Proposition 4.2 we know that $v \leqslant u_{\lambda}$. Then we can define the following Carathéodory function

$$
\hat{k}_{\mu}(z, x)= \begin{cases}v(z)^{-\gamma}+\mu f(z, v(z)) & \text { if } x<v(z) \\ x^{-\gamma}+\mu f(z, x) & \text { if } v(z) \leqslant x \leqslant u_{\lambda}(z) \\ u_{\lambda}(z)^{-\gamma}+\mu f\left(z, u_{\lambda}(z)\right) & \text { if } u_{\lambda}(z)<x\end{cases}
$$

We set $\hat{K}_{\mu}(z, x)=\int_{0}^{x} \hat{k}_{\mu}(z, s) d s$ and consider the $C^{1}$-functional $\hat{\Psi}_{\mu}: W^{1, p}(\Omega) \rightarrow \mathbb{R}$ defined by

$$
\hat{\Psi}_{\mu}(u)=\frac{1}{p} \gamma_{p}(u)+\frac{1}{q}\|D u\|_{q}^{q}-\int_{\Omega} \hat{K}_{\mu}(z, u) d z \text { for all } u \in W^{1, p}(\Omega) .
$$

Evidently, $\hat{\Psi}_{\mu}(\cdot)$ is coercive (see $\left.(25)\right)$ and sequentially weakly lower semicontinuous. So, we can find $u_{\mu} \in W^{1, p}(\Omega)$ such that

$$
\begin{aligned}
& \hat{\Psi}_{\mu}\left(u_{\mu}\right)=\inf \left\{\hat{\Psi}_{\mu}(u): u \in W^{1, p}(\Omega)\right\} \\
\Rightarrow & \hat{\Psi}_{\mu}^{\prime}\left(u_{\mu}\right)=0 \\
\Rightarrow & \left\langle A_{p}\left(u_{\mu}\right), h\right\rangle+\left\langle A_{q}\left(u_{\mu}\right), h\right\rangle+\int_{\Omega} \xi(z)\left|u_{\mu}\right|^{p-2} u_{\mu} h d z+\int_{\partial \Omega} \beta(z)\left|u_{\mu}\right|^{p-2} u_{\mu} h d \sigma \\
= & \int_{\Omega} \hat{k}_{\mu}(z, u \mu) h d z \text { for all } h \in W^{1, p}(\Omega) .
\end{aligned}
$$

In (26) first we choose $h=\left(u_{\mu}-u_{\lambda}\right)^{+} \in W^{1, p}(\Omega)$. Using (25), the fact that $\mu<\lambda$ and that $f \geqslant 0$ and recalling that $u_{\lambda} \in S_{\lambda}$, we conclude that $u_{\mu} \leqslant u_{\lambda}$. Next, in (26) we choose $h=\left(v-u_{\mu}\right)^{+} \in$ $W^{1, p}(\Omega)$. From (25), the fact that $f \geqslant 0$ and Proposition 3.2, we infer that $v \leqslant u_{\mu}$. Therefore we have proved that

$$
u_{\mu} \in\left[v, u_{\lambda}\right]
$$

From (25), (26), (27) it follows that

$$
u_{\mu} \in S_{\mu} \subseteq D_{+}(\text {see Proposition 4.1). }
$$


Let $\rho=\left\|u_{\lambda}\right\|_{\infty}$ and let $\hat{\xi}_{\rho}^{\lambda}>0$ be as postulated by hypothesis $H(f)(v)$. We have

$$
\begin{aligned}
& -\Delta_{p} u_{\lambda}(z)-\Delta_{q} u_{\mu}(z)+\left[\xi(z)+\hat{\xi}_{\rho}^{\lambda}\right] u_{\mu}(z)^{p-1}-u_{\mu}(z)^{-\gamma} \\
= & \mu f\left(z, u_{\mu}(z)\right)+\hat{\xi}_{\rho}^{\lambda} u_{\mu}(z)^{p-1} \\
= & \lambda f\left(z, u_{\mu}(z)\right)+\hat{\xi}_{\rho}^{\lambda} u_{\mu}(z)^{p-1}-(\lambda-\mu) f\left(z, u_{\mu}(z)\right) \\
< & \left.\lambda f\left(z, u_{\mu}(z)\right)+\hat{\xi}_{\rho}^{\lambda} u_{\lambda}(z)^{p-1} \text { (recall that } \lambda>\mu\right) \\
\leqslant & \left.\lambda f\left(z, u_{\lambda}(z)\right)+\hat{\xi}_{\rho}^{\lambda} u_{\lambda}(z)^{p-1} \text { (see }(27) \text { and hypothesis } H(f)(v)\right) \\
= & -\Delta_{p} u_{\lambda}(z)-\Delta_{q} u_{\lambda}(z)+\left[\xi(z)+\hat{\xi}_{\rho}^{\lambda}\right] u_{\lambda}(z)^{p-1}-u_{\lambda}(z)^{-\lambda} \text { for almost all } z \in \Omega \\
& \left(\text { recall that } u_{\lambda} \in S_{\lambda}\right) .
\end{aligned}
$$

We know that

$$
0 \leqslant u_{\mu}^{-\gamma}, u_{\lambda}^{-\gamma} \leqslant v^{-\gamma} \in L^{\infty}(\Omega) .
$$

Also, from hypothesis $H(f)(i v)$ and since $u_{\mu} \in D_{+}$, we have

$$
0<c_{8} \leqslant(\lambda-\mu) f\left(z, u_{\mu}(z)\right) \text { for almost all } z \in \Omega .
$$

Invoking Proposition 2.4, from (28) we conclude that

$$
u_{\lambda}-u_{\mu} \in \operatorname{int} \hat{C}_{+} \text {. }
$$

The proof is now complete.

Proposition 4.4. If hypotheses $H(\xi), H(\beta), H_{0}, H(f)$ hold, then $\lambda^{*}<+\infty$.

Proof. On account of hypotheses $H(f)(i) \rightarrow(i v)$, we can find $\lambda_{0}>0$ big such that

$$
x^{-\gamma}+\lambda_{0} f(z, x) \geqslant x^{p-1} \text { for almost all } z \in \Omega \text { and all } x \geqslant 0 .
$$

Let $\lambda>\lambda_{0}$ and suppose that $\lambda \in \mathcal{L}$. Then we can find $u_{\lambda} \in S_{\lambda} \subseteq D_{+}$(see Proposition 4.1). Then $m_{\lambda}=\min _{\bar{\Omega}} u_{\lambda}>0$. For $\delta \in(0,1)$ we set $m_{\lambda}^{\delta}=m_{\lambda}+\delta$ and for $\rho=\left\|u_{\lambda}\right\|_{\infty}$ let $\hat{\xi}_{\rho}^{\lambda}>0$ be as postulated by hypothesis $H(f)(v)$. We have

$$
\begin{aligned}
& -\Delta_{p} m_{\lambda}^{\delta}-\Delta_{q} m_{\lambda}^{\delta}+\left[\xi(z)+\hat{\xi}_{\rho}\right]\left(m_{\lambda}^{\delta}\right)^{p-1}-\left(m_{\lambda}^{\delta}\right)^{-\gamma} \\
= & {\left[\xi(z)+\hat{\xi}_{\rho}^{\lambda}\right] m_{\lambda}^{p-1}-m_{\lambda}^{-\gamma}+\chi(\delta) \text { with } \chi(\delta) \rightarrow 0^{+} \text {as } \delta \rightarrow 0^{+} } \\
< & \xi(z) m_{\lambda}^{p-1}+\left(1+\hat{\xi}_{\rho}^{\lambda}\right) m_{\lambda}^{p-1}-m_{\lambda}^{-\gamma}+\chi(\delta) \\
\leqslant & \lambda_{0} f\left(z, m_{\lambda}\right)+\left[\xi(z)+\hat{\xi}_{\rho}^{\lambda}\right] m_{\lambda}^{p-1}+\chi(\delta)(\text { see }(29)) \\
\leqslant & \lambda_{0} f\left(z, u_{\lambda}\right)+\left[\xi(z)+\hat{\xi}_{\rho}^{\lambda}\right] u_{\lambda}^{p-1}+\chi(\delta)(\text { see hypothesis } H(f)(v)) \\
= & \lambda f\left(z, u_{\lambda}\right)+\left[\xi(z)+\hat{\xi}_{\rho}^{\lambda}\right] u_{\lambda}^{p-1}-\left(\lambda-\lambda_{0}\right) f\left(z, u_{\lambda}\right)+\chi(\delta) \\
= & \lambda f\left(z, u_{\lambda}\right)+\left[\xi(z)+\hat{\xi}_{\rho}^{\lambda}\right] u_{\lambda}^{p-1} \text { for } \delta \in(0,1) \text { small } \\
& \left(\text { recall that } u_{\lambda} \in D_{+} \text {and see } H(f)(i v)\right) \\
= & -\Delta_{p} u_{\lambda}-\Delta_{q} u_{\lambda}+\left[\xi(z)+\hat{\xi}_{\rho}^{\lambda}\right] u_{\lambda}^{p-1}-u_{\lambda}^{-\gamma} .
\end{aligned}
$$

Since $\left(\lambda-\lambda_{0}\right) f\left(z, u_{\lambda}\right)-\chi(\delta) \geqslant c_{9}>0$ for almost all $z \in \Omega$ and for $\delta \in(0,1)$ small (just recall that $u_{\lambda} \in D_{+}$and use hypothesis $\mathrm{H}(\mathrm{f})$ (iv), invoking Proposition 2.4, from (30) we infer that

$$
u_{\lambda}-m_{\lambda}^{\delta} \in \operatorname{int} \hat{C}_{+} \text {for all } \delta \in(0,1) \text { small enough. }
$$

However, this contradicts the definition of $m_{\lambda}$. It follows that $\lambda \notin \mathcal{L}$ and so $\lambda^{*} \leqslant \lambda_{0}<+\infty$.

Therefore we have

$$
\left(0, \lambda^{*}\right) \subseteq \mathcal{L} \subseteq\left(0, \lambda^{*}\right]
$$

Proposition 4.5. If hypotheses $H(\xi), H(\beta), H_{0}, H(f)$ hold and $\lambda \in\left(0, \lambda^{*}\right)$, then problem $\left(P_{\lambda}\right)$ has at least two positive solutions

$$
u_{0}, \hat{u} \in D_{+}, u_{0} \neq \hat{u} .
$$


Proof. Let $0<\mu<\lambda<\eta<\lambda^{*}$. According to Proposition 4.3, we can find $u_{\eta} \in S_{\eta} \subseteq D_{+}$, $u_{0} \in S_{\lambda} \subseteq D_{+}$and $u_{\mu} \in S_{\mu} \subseteq D_{+}$such that

$$
\begin{aligned}
& u_{\eta}-u_{0} \in \operatorname{int} \hat{C}_{+} \text {and } u_{0}-u_{\mu} \in \operatorname{int} \hat{C}_{+}, \\
& \Rightarrow u_{0} \in \operatorname{int}_{C^{1}(\hat{\Omega})}\left[u_{\mu}, u_{\eta}\right] .
\end{aligned}
$$

We introduce the following Carathéodory function

$$
\tilde{\tau}_{\lambda}(z, x)= \begin{cases}u_{\mu}(z)^{-\gamma}+\lambda f\left(z, u_{\mu}(z)\right) & \text { if } x<u_{\mu}(z) \\ x^{-\gamma}+\lambda f(z, x) & \text { if } u_{\mu}(z) \leqslant x \leqslant u_{\eta}(z) \\ u_{\eta}(z)^{-\gamma}+\lambda f\left(z, u_{\eta}(z)\right) & \text { if } u_{\eta}(z)<x\end{cases}
$$

Set $\tilde{T}_{\lambda}(z, x)=\int_{0}^{x} \tilde{\tau}_{\lambda}(z, s) d s$ and consider the $C^{1}$-functional $\tilde{\Psi}_{\lambda}: W^{1, p}(\Omega) \rightarrow \mathbb{R}$ defined by

$$
\tilde{\Psi}_{\lambda}(u)=\frac{1}{p} \gamma_{p}(u)+\frac{1}{q}\|D u\|_{q}^{q}-\int_{\lambda} \tilde{T}_{\lambda}(z, u) d z \text { for all } u \in W^{1, p}(\Omega) .
$$

Using (32) and the nonlinear regularity theory, we can easily check that

$$
K_{\tilde{\Psi}_{\lambda}} \subseteq\left[u_{\mu}, u_{\eta}\right] \cap D_{+} .
$$

Also, consider the Carathéodory function

$$
\tau_{\lambda}^{*}(z, x)= \begin{cases}u_{\mu}(z)^{-\gamma}+\lambda f\left(z, u_{\mu}(z)\right) & \text { if } x \leqslant u_{\mu}(z) \\ x^{-\gamma}+\lambda f(z, x) & \text { if } u_{\mu}(z)<x .\end{cases}
$$

We set $T_{\lambda}^{*}(z, x)=\int_{0}^{x} \tau_{\lambda}^{*}(z, s) d s$ and consider the $C^{1}$-functional $\Psi_{\lambda}^{*}: W^{1, p}(\Omega) \rightarrow \mathbb{R}$ defined by

$$
\Psi_{\lambda}^{*}(u)=\frac{1}{p} \gamma_{p}(u)+\frac{1}{q}\|D u\|_{q}^{q}-\int_{\Omega} T_{\lambda}^{*}(z, u) d z \text { for all } u \in W^{1, p}(\Omega) .
$$

For this functional using (34), we show that

$$
K_{\Psi_{\lambda}^{*}} \subseteq\left[u_{\mu}\right) \cap D_{+} .
$$

From (32) and (34) we see that

$$
\left.\tilde{\Psi}_{\lambda}\right|_{\left[u_{\mu}, u_{\eta}\right]}=\left.\Psi_{\lambda}^{*}\right|_{\left[u_{\mu}, u_{\eta}\right]} \text { and }\left.\tilde{\Psi}_{\lambda}^{\prime}\right|_{\left[u_{\mu}, u_{\eta}\right]}=\left.\left(\Psi_{\lambda}^{*}\right)^{\prime}\right|_{\left[u_{\mu}, u_{\lambda}\right]} .
$$

From (33), (35), (36), it follows that without any loss of generality, we may assume that

$$
K_{\Psi_{\lambda}^{*}} \cap\left[u_{\mu}, u_{\eta}\right]=\left\{u_{0}\right\} .
$$

Otherwise it is clear from (34) and (35) that we already have a second positive smooth solution for problem $\left(P_{\lambda}\right)$ and so we are done.

Note that $\tilde{\Psi}_{\lambda}(\cdot)$ is coercive (see (32)). Also, it is sequentially weakly lower semicontinuous. So, we can find $\hat{u}_{0} \in W^{1, p}(\Omega)$ such that

$$
\begin{aligned}
& \tilde{\Psi}_{\lambda}\left(\hat{u}_{0}\right)=\inf \left\{\tilde{\Psi}_{\lambda}(u): u \in W^{1, p}(\Omega)\right\}, \\
& \Rightarrow \hat{u}_{0} \in K_{\tilde{\Psi}_{\lambda}}, \\
& \Rightarrow \hat{u}_{0} \in K_{\Psi_{\lambda}^{*}} \cap\left[u_{\mu}, u_{\eta}\right](\text { see }(33),(36)), \\
& \Rightarrow \hat{u}_{0}=u_{0} \in D_{+}(\text {see }(37)), \\
& \Rightarrow u_{0} \text { is a local } C^{1}(\bar{\Omega}) \text {-minimizer of } \Psi_{\lambda}^{*}(\text { see }(31)), \\
& \left.\Rightarrow u_{0} \text { is a local } W^{1, p}(\Omega) \text {-minimizer of } \Psi_{\lambda}^{*} \text { (see Proposition } 2.5\right) .
\end{aligned}
$$

We assume that $K_{\Psi_{\lambda}^{*}}$ is finite. Otherwise on account of (34) and (35) we see that we already have an infinity of positive smooth solutions for problem $\left(P_{\lambda}\right)$ and so we are done. Then (38) implies that we can find $\rho \in(0,1)$ small. such that 


$$
\Psi_{\lambda}^{*}\left(u_{0}\right)<\inf \left\{\Psi_{\lambda}^{*}(u):\left\|u-u_{0}\right\|=\rho\right\}=m_{\lambda}^{*}
$$

(see Papageorgiou, Rădulescu \& Repovš [15, Theorem 5.7.6, p. 367]).

On account of hypothesis $H(f)(i i)$ we have

$$
\Psi_{\lambda}^{*}\left(t \hat{u}_{1}(p)\right) \rightarrow-\infty \text { as } t \rightarrow+\infty .
$$

Claim 1. $\Psi_{\lambda}^{*}(\cdot)$ satisfies the $C$ - condition.

Let $\left\{u_{n}\right\}_{n \geqslant 1} \subseteq \mathrm{W}^{1, p}(\Omega)$ be a sequence such that

$$
\begin{gathered}
\left|\Psi_{\lambda}^{*}\left(u_{n}\right)\right| \leqslant c_{10} \text { for some } c_{10}>0 \text { and all } n \in \mathbb{N}, \\
\left(1+\left\|u_{n}\right\|\right)\left(\Psi_{\lambda}^{*}\right)^{\prime}\left(u_{n}\right) \rightarrow 0 \text { in } \mathrm{W}^{1, p}(\Omega)^{*} .
\end{gathered}
$$

From (42) we have

$$
\begin{aligned}
& \left.\left|\left\langle A_{p}\left(u_{n}\right), h\right\rangle+\left\langle A_{q}\left(u_{n}\right), h\right\rangle+\int_{\Omega} \xi(z)\right| u_{n}\right|^{p-2} u_{n} h d z+\int_{\partial \Omega} \beta(z)\left|u_{n}\right|^{p-2} u_{n} h d \sigma \\
& -\int_{\Omega} \tau_{\lambda}^{*}\left(z, u_{n}\right) h d z \mid \leqslant \frac{\epsilon_{n}|| h \|}{1+\left\|u_{n}\right\|} \text { for all } h \in W^{1, p}, \text { with } \epsilon_{n} \rightarrow 0^{+} .
\end{aligned}
$$

Choosing $h=-u_{n}^{-} \in W^{1, p}(\Omega)$, we obtain

$$
\gamma_{p}\left(u_{n}^{-}\right)+\left\|D u_{n}^{-}\right\|_{q}^{q} \leqslant c_{11}\left\|u_{n}^{-}\right\| \text {for some } c_{11}>0 \text { and all } n \in \mathbb{N} \text { (see (34)) }
$$

$$
\Rightarrow\left\{u_{n}^{-}\right\}_{n \geqslant 1} \subseteq W^{1, p}(\Omega) \text { is bounded (see (1) and recall that } 1<p \text { ). }
$$

Next in (43) we choose $h=u_{n}^{+} \in W^{1, p}(\Omega)$. Then

$$
\begin{aligned}
& -\gamma_{p}\left(u_{n}^{+}\right)-\left\|D u_{n}^{+}\right\|_{q}^{q}+\int_{\Omega} \tau_{\lambda}^{*}\left(z, u_{n}\right) u_{n}^{+} d z \leqslant \epsilon_{n} \text { for all } n \in \mathbb{N}, \\
& \Rightarrow-\gamma_{p}\left(u_{n}^{+}\right)-\left\|D u_{n}^{+}\right\|_{q}^{q}+\int_{\left\{u_{n} \leqslant u_{\mu}\right\}}\left[u_{\mu}^{-\gamma}+\lambda f\left(z, u_{\mu}\right)\right] u_{n}^{+} d z \\
& +\int_{\left\{u_{\mu}<u_{n}\right\}}\left[u_{n}^{-\gamma}+\lambda f\left(z, u_{n}\right)\right] u_{n}^{+} d z \leqslant \epsilon_{n} \text { for all } n \in \mathbb{N} \text { (see (34)) }
\end{aligned}
$$

On the other hand from (41) and (44), we have

$$
\begin{aligned}
& \gamma_{p}\left(u_{n}^{+}\right)+\frac{p}{q}\left\|D u_{n}^{+}\right\|_{q}^{q}-\int_{\left\{u_{n} \leqslant u_{\mu}\right\}} p\left[u_{\mu}^{-\gamma}+\lambda f\left(z, u_{p}\right)\right] u_{n}^{+} d z \\
& -\int_{\left\{u_{\mu}<u_{n}\right\}}\left[\frac{p}{1-\gamma}\left(u_{n}^{1-\gamma}-u_{\mu}^{1-\gamma}\right)+p\left(\lambda F\left(z, u_{n}\right)-\lambda F\left(z, u_{\mu}\right)\right)\right] d z \leqslant \epsilon_{n} \\
& \text { for all } n \in \mathbb{N}(\text { see }(34)) .
\end{aligned}
$$$$
\Rightarrow \gamma_{p}\left(u_{n}^{+}\right)+\frac{p}{q}\left\|D u_{n}^{+}\right\|_{p}^{p}-\int_{\left\{u_{n} \leqslant u_{\mu}\right\}} p\left[u_{\mu}^{-\gamma}+\lambda f\left(z, u_{\mu}\right)\right] u_{n}^{+} d z
$$$$
-\int_{\left\{u_{p}<u_{n}\right\}}\left[\frac{p}{1-\gamma} u_{n}^{1-\gamma}+\lambda p F\left(z, u_{n}\right)\right] d z \leqslant c_{12} \text { for some } c_{12}>0 \text { and all } n \in \mathbb{N} .
$$

We add (45) and (46). Since $p>q$, we obtain

$$
\begin{aligned}
& \lambda \int_{\left\{u_{\mu}<u_{n}\right\}}\left[f\left(z, u_{n}\right) u_{n}^{+}-p F\left(z, u_{n}\right)\right] d z \leqslant(p-1) \int_{\left\{u_{n} \leqslant u_{\mu}\right\}}\left[u_{\mu}^{-\gamma}+\lambda f\left(z, u_{\mu}\right)\right] u_{n}^{+} d z \\
& +\left(\frac{p}{1-\gamma}-1\right) \int_{\left\{u_{\mu}<u_{n}\right\}} u_{n}^{1-\gamma} d z \\
\Rightarrow & \lambda \int_{\Omega}\left[f\left(z, u_{n}^{+}\right) u_{n}^{+}-p F\left(z, u_{n}^{+}\right)\right] d z \leqslant c_{13}\left[\left\|u_{n}^{+}\right\|_{1}+1\right] \\
& \text { for some } c_{13}>0, \text { all } n \in \mathbb{N} .
\end{aligned}
$$


On account of hypotheses $H(f)(i),($ iii $)$ we can find $\hat{\beta}_{1} \in\left(0, \hat{\beta}_{0}\right)$ and $c_{14}>0$ such that

$$
\hat{\beta}_{1} x^{\tau}-c_{14} \leqslant f(z, x)-p F(z, x) \text { for almost all } z \in \Omega \text { and all } x \geqslant 0 .
$$

Using (48) in (47), we obtain

$$
\begin{gathered}
\left\|u_{n}^{+}\right\|_{\tau}^{\tau} \leqslant c_{15}\left[\left\|u_{n}^{+}\right\|_{\tau}+1\right] \text { for some } c_{15}>0 \text { and all } n \in \mathbb{N} \\
\Rightarrow\left\{u_{n}^{+}\right\}_{n \geqslant 1} \leqslant L^{\tau}(\Omega) \text { is bounded. }
\end{gathered}
$$

First assume $N \neq p$. From hypothesis $H(f)($ iii $)$ it is clear that we may assume without any loss of generality that $\tau<r<p^{*}$. Let $t \in(0,1)$ be such that

$$
\frac{1}{r}=\frac{1-t}{\tau}+\frac{t}{p *} .
$$

Then from the interpolation inequality (see Papageorgiou \& Winkert [19, Proposition 2.3.17, p. 116]), we have

$$
\begin{aligned}
&\left\|u_{n}^{+}\right\|_{r} \leqslant\left\|u_{n}^{+}\right\|_{\tau}^{1-t}\left\|u_{n}^{+}\right\|_{p^{*}}^{t}, \\
& \Rightarrow \quad\left\|u_{n}^{+}\right\|_{r}^{r} \leqslant c_{16}\left\|u_{n}^{+}\right\| \|^{t r} \text { for some } c_{16}>0 \text { and all } n \in \mathbb{N}(\text { see }(49)) .
\end{aligned}
$$

From hypothesis $H(f)(i)$ we have

$$
f(z, x) x \leqslant c_{17}\left[1+x^{r}\right] \text { for all } z \in \Omega \text {, all } x \geqslant 0 \text { and some } c_{17}>0 .
$$

From (43) with $h=u_{n}^{+} \in W^{1, p}(\Omega)$, we obtain

$$
\begin{aligned}
& \gamma_{p}\left(u_{n}^{+}\right)+\left\|D u_{n}^{+}\right\|_{q}^{q}-\int_{\Omega} \tau_{\lambda}^{*}\left(z, u_{n}\right) u_{n}^{+} d z \leqslant \epsilon_{n} \text { for all } n \in \mathbb{N}, \\
\Rightarrow & \gamma_{p}\left(u_{n}^{+}\right)+\left\|D u_{n}^{+}\right\|_{q}^{q} \leqslant \int_{\Omega}\left[\left(u_{n}^{+}\right)^{1-\gamma}+f\left(z, u_{n}^{+}\right) u_{n}^{+}\right] d z+c_{18} \\
& \text { for some } c_{18}>0 \text { and all } n \in \mathbb{N}(\text { see }(34)) \\
\leqslant & c_{19}\left[1+\left\|u_{n}^{+}\right\|_{r}^{r}\right] \text { for some } c_{19}>0 \text { and all } n \in \mathbb{N}(\text { see }(51)) \\
\leqslant & c_{20}\left[1+\left\|u_{n}^{+}\right\|^{t r}\right] \text { for some } c_{20}>0 \text { and all } n \in \mathbb{N}(\text { see }(50)) .
\end{aligned}
$$

The hypothesis on $\tau$ (see $H(f)(i i i))$ implies that $t r<p$. So, from (52) we infer that

$$
\begin{gathered}
\left\{u_{n}^{+}\right\}_{n \geqslant 1} \subseteq W^{1, p}(\Omega) \text { is bounded, } \\
\Rightarrow\left\{u_{n}\right\}_{n \geqslant 1} \subseteq W^{1, p}(\Omega) \text { is bounded (see (44)). }
\end{gathered}
$$

If $N=p$, then $p^{*}=+\infty$ and from the Sobolev embedding theorem, we know that $W^{1, p}(\Omega) \hookrightarrow$ $L^{s}(\Omega)$ for all $1 \leqslant s<\infty$. Then in order for the previous argument to work, we replace $p^{*}=+\infty$ by $s>r>\tau$ and let $t \in(0,1)$ as before such that

$$
\begin{aligned}
& \frac{1}{r}=\frac{1-t}{\tau}+\frac{t}{s}, \\
& \Rightarrow t r=\frac{s(r-\tau)}{s-\tau} .
\end{aligned}
$$

Note that $\frac{s(r-\tau)}{s-\tau} \rightarrow r-\tau$ as $s \rightarrow+\infty$. But $r-\tau<p$ (see hypothesis H(f)(iii)). We choose $s>r$ big so that $t r<p$. Then again we have (53).

Because of (53) and by passing to a subsequence if neccesary, we may assume that

$$
u_{n} \stackrel{w}{\rightarrow} u \text { in } W^{1, p}(\Omega) \text { and } u_{n} \rightarrow u \text { in } L^{r}(\Omega) \text { and } L^{p}(\partial \Omega) .
$$

In (43) we choose $h=u_{n}-u \in W^{1, p}(\Omega)$, pass to the limit as $n \rightarrow \infty$ and use (54). Then

$$
\lim _{n \rightarrow \infty}\left[\left\langle A_{p}\left(u_{n}\right), u_{n}-u\right\rangle+\left\langle A_{q}\left(u_{n}\right), u_{n}-u\right\rangle\right]=0,
$$




$$
\begin{aligned}
& \Rightarrow \limsup _{n \rightarrow \infty}\left[\left\langle A_{p}\left(u_{n}\right), u_{n}-u\right\rangle+\left\langle A_{q}(u), u_{n}-u\right\rangle\right] \leqslant 0 \\
& \text { (since } A_{q}(\cdot) \text { is monotone) } \\
& \Rightarrow \limsup _{n \rightarrow \infty}\left\langle A_{p}\left(u_{n}\right), u_{n}-u\right\rangle \leqslant 0, \\
& \Rightarrow u_{n} \rightarrow u \text { in } W^{1, p}(\Omega) \text { (see Proposition 2.1). }
\end{aligned}
$$

Therefore $\Psi_{\lambda}^{*}(\cdot)$ satisfies the C-condition. This proves the claim.

Then (39), (40) and Claim permit the use of the mountain pass theorem. So, we can find $\hat{u} \in W^{1, p}(\Omega)$ such that

$$
\hat{u} \in K_{\Psi_{\lambda}^{*}} \leqslant\left[u_{\mu}\right) \cap D_{+}(\text {see }(35)), m_{\lambda}^{*} \leqslant \Psi_{\lambda}^{*}(\hat{u})(\text { see }(39)) .
$$

Therefore $\hat{u} \in D_{+}$is a second positive solution of problem $\left(P_{\lambda}\right)\left(\lambda \in\left(0, \lambda^{*}\right)\right)$ distinct from $u_{0} \in D_{+}$.

Next, we examine what can be said in the critical parameter $\lambda^{*}$.

Proposition 4.6. If hypotheses $H(\xi), H(\beta), H_{0}, H(f)$ hold, then $\lambda^{*} \in \mathcal{L}$.

Proof. Let $\left\{\lambda_{n}\right\}_{n \geqslant 1} \subseteq\left(0, \lambda^{*}\right)$ be such that $\lambda_{n}<\lambda^{*}$. We can find $u_{n} \in S_{\lambda_{n}} \subseteq D_{+}$for all $n \in \mathbb{N}$.

We consider the following Carathéodory function

$$
\mu_{n}(z, x)= \begin{cases}v(z)^{-\gamma}+\lambda_{n} f(z, v(z)) & \text { if } x \leqslant v(z) \\ x^{-\gamma}+\lambda_{n} f(z, x) & \text { if } v(z)<x .\end{cases}
$$

We set $M_{n}(z, x)=\int_{0}^{x} \mu_{n}(z, x) d s$ and consider the $C^{1}$-functional $j_{n}: W^{1, p}(\Omega) \rightarrow \mathbb{R}$ defined by

$$
j_{n}(u)=\frac{1}{p} \gamma_{p}(u)+\frac{1}{q}\|D u\|_{q}^{q}-\int_{\Omega} M_{n}(z, u) d z \text { for all } u \in W^{1, p}(\Omega) .
$$

Also, we consider the following truncation of $\mu_{n}(z, \cdot)$

$$
\hat{\mu}_{n}(z, x)= \begin{cases}\mu_{n}(z, x) & \text { if } x \leqslant u_{n+1}(z) \\ \mu_{n}\left(z, u_{n+1}(z)\right) & \text { if } u_{n+1}(z)<x\end{cases}
$$

(recall that $v \leqslant u_{n+1}$ for all $n \in \mathbb{N}$, see Proposition 4.2). This is a Carathéodory function. We set $\hat{M}_{n}(z, x)=\int_{0}^{x} \hat{\mu}_{n}(z, s) d s$ and consider the $C^{1}$-functional $\hat{J}_{n}: W^{1, p}(\Omega) \rightarrow \mathbb{R}$ defined by

$$
\hat{J}_{n}(u)=\frac{1}{p} \gamma_{p}(u)+\frac{1}{q}\|D u\|_{q}^{q}-\int_{\Omega} \hat{M}_{n}(z, u) d z \text { for all } u \in W^{1, p}(\Omega) .
$$

From (55), (56) and (1), it is clear that $\hat{J}_{n}(\cdot)$ is coercive. Also, it is sequentially weakly lower semicontinuous. So, we can find $\hat{u}_{n} \in W^{1, p}(\Omega)$ such that

$$
\hat{J}_{n}\left(\hat{u}_{n}\right)=\inf \left\{\hat{J}_{n}(u): u \in W^{1, p}(\Omega)\right\} .
$$

Then we have

$$
\begin{aligned}
\hat{J}_{n}\left(\hat{u}_{n}\right) \leqslant & \hat{J}_{n}(v) \\
\leqslant & \frac{1}{p} \gamma_{p}(v)+\frac{1}{q}\|D v\|_{q}^{q}-\frac{1}{1-\gamma} \int_{\Omega} v^{1-\gamma} d z \\
& (\operatorname{see}(55),(56) \text { and recall that } f \geqslant 0) \\
\leqslant & \left\langle A_{p}(v), v\right\rangle+\left\langle A_{q}(v), v\right\rangle-\int_{\Omega} v^{1-\gamma} d z=0
\end{aligned}
$$

(see Proposition 3.2).

From (57) we have

$$
\hat{u}_{n} \in K_{\hat{J}_{n}} \subseteq\left[v, u_{n+1}\right] \cap D_{+} \text {for all } n \in \mathbb{N}(\text { see }(56)) .
$$

Similarly, using (55) we obtain

$$
K_{j_{n}} \subseteq[v) \cap D_{+} .
$$


Note that

$$
\left.J_{n}\right|_{\left[v, u_{n+1}\right]}=\left.\hat{J}_{n}\right|_{\left[v, u_{n+1}\right]} \text { and }\left.J_{n}^{\prime}\right|_{\left[v, u_{n+1}\right]}=\left.\hat{J}_{n}^{\prime}\right|_{\left[v, u_{n+1}\right]}(\operatorname{see}(55),(56)) .
$$

Then from (58), (59), (60), we have

$$
\begin{aligned}
& \left\langle A_{p}\left(\hat{u}_{n}\right), h\right\rangle+\left\langle A_{q}\left(\hat{u}_{n}\right), h\right\rangle+\int_{\Omega} \xi(z) \hat{u}_{n}^{p-1} h d z+\int_{\partial \Omega} \beta(z) \hat{u}_{n}^{p-1} h d \sigma=\int_{\Omega} \mu_{n}\left(z, \hat{u}_{n}\right) h d z \\
& \quad \text { for all } h \in W^{1, p}(\Omega), \text { all } n \in \mathbb{N} .
\end{aligned}
$$

Using (61), (62) and reasoning as in the Claim in the proof of Proposition 4.5, we show that

$$
\left\{\hat{u}_{n}\right\}_{n \geqslant 1} \subseteq W^{1, p}(\Omega) \text { is bounded. }
$$

So, we may assume that

$$
\hat{u}_{n} \stackrel{w}{\rightarrow} \hat{u}_{*} \text { in } W^{1, p}(\Omega) \text { and } \hat{u}_{n} \rightarrow \hat{u}_{*} \text { in } L^{r}(\Omega) \text { and } L^{p}(\partial \Omega) .
$$

In (62) we choose $h=\hat{u}_{n}-\hat{u}_{*} \in W^{1, p}(\Omega)$, pass to the limit as $n \rightarrow \infty$ and use (63). Then as before (see the proof of Proposition 4.5), we obtain

$$
\hat{u}_{n} \rightarrow \hat{u}_{*} \text { in } W^{1, p}(\Omega) .
$$

In (62) we pass to the limit as $n \rightarrow \infty$ and use (64). Then

$$
\begin{gathered}
\left\langle A_{p}\left(\hat{u}_{*}\right), h\right\rangle+\left\langle A_{q}\left(\hat{u}_{*}\right), h\right\rangle+\int_{\Omega} \xi(z) \hat{u}_{*}^{p-1} h d z+\int_{\partial \Omega} \beta(z) \hat{u}_{*}^{p-1} h d z \\
=\int_{\Omega}\left[\hat{u}_{*}^{-\gamma}+\lambda^{*} f\left(z, \hat{u}_{*}\right)\right] h d z \text { for all } h \in W^{1, p}(\Omega)(\text { see }(55),(60)), \\
\Rightarrow \hat{u}_{*} \in S_{\lambda^{*}} \subseteq D_{+} \text {and so } \lambda^{*} \in \mathcal{L} .
\end{gathered}
$$

The proof is now complete.

From this proposition it follows that

$$
\mathcal{L}=(0, \lambda *]
$$

The next bifurcation-type theorem summarizes our findings and provides a complete description of the dependence of the set of positive solutions of problem $\left(P_{\lambda}\right)$ on the parameter $\lambda>0$.

Theorem 4.7. If hypotheses $H(\xi), H(\beta), H_{0}, H(f)$ hold, then there exists $\lambda^{*}>0$ such that

(a) for all $\lambda \in\left(0, \lambda^{*}\right)$ problem $\left(P_{\lambda}\right)$ has at least two positive solutions

$$
u_{0}, \hat{u} \in D_{+}, u_{0} \neq \hat{u}
$$

(b) for $\lambda=\lambda^{*}$ problem $\left(P_{\lambda}\right)$ has at least one positive solution $\hat{u}_{*} \in D_{+}$;

(c) for all $\lambda>\lambda^{*}$ problem $\left(P_{\lambda}\right)$ does not have any positive solutions.

\section{ACKNOWLEDGEMENTS.}

This research was supported by the Slovenian Research Agency grants P1-0292, J1-8131, J1-7025, N1-0064, and N1-0083. 


\section{REFERENCES}

[1] A. Bahrouni, V.D. Rădulescu, D.D. Repovš, Double-phase transonic flow problems with variable growth: nonlinear patterns and stationary waves, Nonlinearity 32 (2019), no. 7, 2481-2495.

[2] L. Cherfils, Y. Ilyasov, On the stationary solutions of generalized reaction-diffusion equations with $p \& q$ Laplacian, Commun. Pure. Appl. Anal. 4 (2005), 9-22.

[3] L. Gasinski, N.S. Papageorgiou, Nonlinear Analysis, Chapman \& Hall/CRC, Boca Raton, FL, 2006.

[4] L. Gasinski, N.S. Papageorgiou, Exercises in Analysis. Part 2: Nonlinear Analysis, Springer, Cham, 2016.

[5] M. Ghergu, V.D. Rădulescu, Singular Elliptic Problems. Bifurcation and Asymptotic Analysis, Oxford Lecture Series in Mathematics and its Applications, vol. 37, The Clarendon Press, Oxford University Press, Oxford, 2008.

[6] J. Giacomoni, J. Schindler, P. Takač, Sobolev versus Hölder local minimizers and existence of multiple solutions for a singular quasilinear equation, Ann. Scuola Normale Super. Pisa, Ser. V 6 (2007), 117-158.

[7] G. Lieberman, The natural generalization of the natural conditions of Ladyzhenskaya and Uraltseva for elliptic equations, Commun. Partial Diff. Equations 16 (1991), 311-361.

[8] N.S. Papageorgiou, V.D. Rădulescu, Multiple solutions with precise sign for nonlinear parametre Robin problems, J. Differential Equations 254 (2014), 393-430.

[9] N.S. Papageorgiou, V.D. Rădulescu, Nonlinear nonhomogeneous Robin problems with superlinear reaction, Adv. Nonlinear Stud. 16 (2016), 737-764.

[10] N.S. Papageorgiou, V.D. Rădulescu, Positive solutions for nonlinear nonhomogeneous parametric Robin problems, Forum Math. 30 (2018), 553-580.

[11] N.S. Papageorgiou, V.D. Rădulescu, D.D. Repovš, Positive solutions for nonlinear parametric singular Dirichlet problems, Bull. Math. Sci. 9 (2019), No. 2, art. 1950011, 21 pp.

[12] N.S. Papageorgiou, V.D. Rădulescu, D.D. Repovš, Pairs of positive solutions for resonant singular equations with the $p$-Laplacian, Electr. J. Diff. Equ. 2017, art. 249, 13 pp.

[13] N.S. Papageorgiou, V.D. Rădulescu, D.D. Repovš, Double-phase problems with reaction of arbitrary growth, $Z$. Angew. Math. Phys. 69 (2018), no. 4, art. 108, 21 pp.

[14] N.S. Papageorgiou, V.D. Rădulescu, D.D. Repovš, Double-phase problems and a discontinuity property of the spectrum, Proc. Amer. Math. Soc. 147 (2019), no. 7, 2899-2910.

[15] N.S. Papageorgiou, V.D. Rădulescu, D.D. Repovš, Modern Nonlinear Analysis - Theory and Methods, Springer, Cham, 2019.

[16] N.S. Papageorgiou, V.D. Rădulescu, D.D. Repovš, Existence and multiplicity of solutions for double-phase Robin problems, Bull. London Math. Soc. 52 (2020), 546-560.

[17] N.S. Papageorgiou, G. Smyrlis, A bifurcation-type theorem for singular nonlinear elliptic equations, Methods Appl. Anal. 22 (2015), 147-170.

[18] N.S. Papageorgiou, P. Winkert, Singular $p$-Laplacian equations with superlinear perturbation, J. Differential Equations 266 (2019), 1462-1487.

[19] N.S. Papageorgiou, P. Winkert, Applied Nonlinear Functional Analysis, de Gruyter, Berlin, 2018.

[20] K. Perera, Z. Zhang, Multiple positive solutions of singular $p$-Laplacian problems by variational methods, Bound. Value Probl. 2005:3, 2005.

[21] P. Pucci, J. Serrin, The Maximum Principle, Birkhäuser, Basel, 2007.

[22] V. V. Zhikov, Averaging of functionals of the calculus of variations and elasticity theory, Math. USSR-Izv. 29 (1987), 33-66.

(N.S. Papageorgiou) Institute of Mathematics, Physics and Mechanics, 1000 Ljubljana, Slovenia \& National Technical University, Zografou Campus, 15780 Athens, Greece

Email address: npapg@math.ntua.gr

(V.D. Rădulescu) Institute of Mathematics, Physics and Mechanics, 1000 Ljubljana, Slovenia \& AGH University of Science and Technology, 30-059 Kraków, Poland

Email address: vicentiu.radulescu@imfm.si

(D.D. Repovš) Faculty of Education and Faculty of Mathematics and Physics, University of Ljubljana \& Institute of Mathematics, Physics and Mechanics, 1000 Ljubluana, Slovenia

Email address: dusan.repovs@guest.arnes.si 